\title{
Coupled fluid-dynamical and structural analysis of a mono-axial mems accelerometer
}

\author{
Cammarata A. and Petrone G.* \\ Department of Industrial Engineering - University of Catania \\ Viale Andrea Doria, 6 - 95126 Catania, ITALY \\ acamma@dii.unict.it
}

\begin{abstract}
This study is aimed to numerically investigate the elastodynamics of a mono-axial MEMS accelerometer. The vibrating part of the device is dipped into a fluid micro-channel and made of a proof mass connected to the frame by two flexible legs. The adopted mathematical model lies on a linearized motion equations system, where the mass matrix is obtained by means of both lumped and distributed approach. The stiffness matrix is otherwise derived through FEA, in which the proof mass and the compliant legs are modeled as rigid and flexible bodies, respectively. The squeezed-film damping effect is evaluated by a fluid-dynamical FE model based on a modified Reynolds formulation. The ensuing analyses are carried-out for three pressure levels of the narrow gas film surrounding the device, by applying the logarithmic decrement method for evaluating the damping ratio. Numerical results, in terms of acceleration, frequency range and noise disturbance, are successfully compared to analytical and experimental ones previously published in literature. Our model characterizes the accelerometer dynamics in space, allowing, in addition, to assess translational motion errors along directions apart the working one.
\end{abstract}

Keywords: MEMS accelerometer, elastodynamics, micro-channel

\section{INTRODUCTION}

The architecture of a compliant mechanism is often obtained by the union of different instances of a same simpler structure rotated and translated in space. In recent years this class of mechanisms spread in industry because of its ease in manufacturing and assembling. Flexure mechanisms are one of the main outcome of this fast diffusion. Their architecture is often monolithic and composed of repeated modules arranged in series or parallel. Flexures are compliant structures that rely on material elasticity to provide smooth motion guidance [1], load bearing capability and force transmission: the application of input forces generates output motion thanks to the deformation of the material. Unlike traditional linkages, flexure mechanisms are not an assembly of different bodies coupled by means of joints, but they are fabricated as a monolithic structure not affected by backlash or friction [2]. Besides, low hysteresis and zero maintenance, due to the absence of wear, make flexure mechanisms well-suited for: M.E.M.S., N.E.M.S.,

${ }^{\star}$ Corresponding author. E-mail: gpetrone@dii.unict.it 
piezoelectric actuators, sensors, positioning and motion systems and many others applications requiring miniaturized systems.

In despite of all the said advantages, the flexure mechanisms still suffer from some limitations such as: small range of motion, variable stiffness along load and motion directions. Where the former limit is often overcome due to the application to micro- and nano-systems, the latter introduces error motions affecting precision and accuracy. Especially for planar flexures, undesired cross-axis and parasitic error motions are often limited by changing geometry, recurring to symmetric layout, or repositioning the loads. Exploiting the same strategies to spatial systems is often more difficult and hard to predict.

Another important requirement is tied to dynamic stability and dynamics response of flexure mechanisms to be used in MEMS applications as: Accelerometers, STEM (Scanning Transmission Electron Microscope), AFM (Atomic Force Microscope) or SPM (Scanning Probe Microscope).

Many studies have been carried out to investigate the dynamics of flexure mechanisms. From classic papers by Simo and Vu-Quoc $[3,4]$ on large rotation vector method, now referred to as Absolute Coordinate Nodal Formulation (ANCF), [3, 4], to co-rotational formulations as the Floating Frame of Reference Formulation (FFRF), [5], most of the research is now based on techniques that simplify the large-deflection nonlinear analysis of flexure mechanism. As instance, the Pseudo-Rigid Body (PRB) models introduce lumped springs and rigid bodies in order to recur to the existing theory of rigid body mechanisms, $[6,7,8]$. Other papers are focused on the well- established FEA theory, $[9,10]$, while some authors recurred to other techniques as the generalized multiple shooting method (GMSM) in which dynamic equations with joint boundary conditions are derived by using Hamilton's principle and are solved by treating a boundary value problem as an initial value problem, [11]. In [12] the authors used Hamilton's principle combined with Newmark scheme to describe the dynamic model of a cantilever, which accounts for bending, shear and axial deformations with no geometric approximation.

In this paper we recur to a linear formulation to study the coupled fluid-dynamical and structural analysis of a mono-axial MEMS accelerometer. This particular MEMs is a planar flexure mechanism used to detect acceleration along its axial direction. As already pointed out in [14], we use the assumption of small displacements, since these "tend to affect important quantities such as air friction, heat dissipation, and electrostatic forces but generally have little effect on the inertia and stiffness properties of the mechanism".

Combining concepts derived from the Matrix Structural Analysis [13] with substructuring techniques the elastodynamics equations of the system are first obtained. Lumped and distributed formulations for the mass matrix determination are then investigated. In order to consider the damping effect coming from the fluid-structure coupling, FEM simulations are performed recurring to a modified Reynolds equation approach that allows to keep into account the damping effect due to squeezed-film and slide-film as well. The damping coefficients and the ensuing damping matrices for three levels of pressure through the use of the Logarithmic Decrement Method are obtained. In the last section numerical simulations are carried out and compared to previous results appearing in literature for validation.

The aim of our work is to yield a simple and practical tool to analyze a MEMs mechanism predicting its motion errors and its dynamic behavior considering different designs or new operative conditions. 


\section{PROBLEM FORMULATION}

\subsection{DEVICE DESCRIPTION}

The geometry of the studied system is reported in Fig. 1. It is made by two main parts, the first one, outlined in the upper portion of the figure, is the mass proof that is linked to the mainframe by two flexible lamina springs located at its double-end. The central anchor body is equipped by several lateral blades that work as electrodes during the devise functioning. The second part is the mainframe, recognizable in the lower portion of the Fig. 1. It is made by a ribbed baseplate where the previously described component is arranged in. Geometrical and physical properties are listed in Table I. The constitutive material of the MEMS is a poly-silicon.

The geometrical coupling between the central body blades and the baseplate ribs determinates several microchannels filled by air. Micro-channels are filled by air kept at a chosen pressure. For that reason, when the proof mass moves it interacts with the air-filled micro-channels, yielding to a dissipation of the vibration. Because of the central body

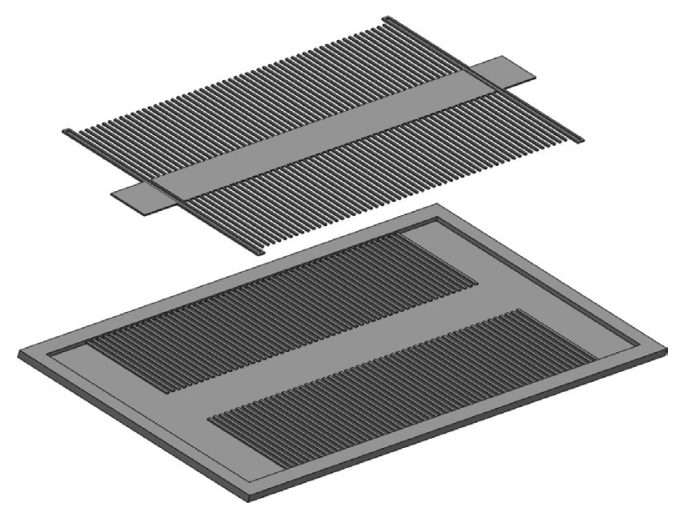

Figure 1 Geometry of the accelerometer.

Table I Geometrical and physical properties.

\begin{tabular}{cccc} 
Symbol & Description & Value & Unit \\
\hline$L$ & Length of the mass & 500 & {$[\mu \mathrm{m}]$} \\
\hline$l$ & Length of the blade & 120 & {$[\mu \mathrm{m}]$} \\
\hline$a$ & Length of the electrode & 110 & {$[\mu \mathrm{m}]$} \\
\hline$w$ & Width of the mass & 50 & {$[\mu \mathrm{m}]$} \\
\hline$b$ & Length of the blade & 10 & {$[\mu \mathrm{m}]$} \\
\hline$h$ & Thickness of the blade & 2.5 & {$[\mu \mathrm{m}]$} \\
\hline$t$ & Thickness of the electrode & 2 & {$[\mu \mathrm{m}]$} \\
\hline$n$ & Number of electrodes & 54 & {$[-]$} \\
\hline$E$ & Young's Modulus & 160 & {$[\mathrm{GPa}]$} \\
\hline$v$ & Poisson's ratio & 0,22 & {$[-]$} \\
\hline$P$ & Material density & 2320 & {$\left[\mathrm{~kg} / \mathrm{m}^{3}\right]$} \\
\hline $\mathrm{V}_{1}$ & Volume of the mass & $1.07 \mathrm{E}-15$ & {$\left[\mathrm{~m}^{3}\right]$} \\
\hline $\mathrm{V}_{2}$ & Volume of the blades & $2.70 \mathrm{E}-15$ & {$\left[\mathrm{~m}^{3}\right]$} \\
\hline
\end{tabular}


stiffness along the longitudinal axis is much lower than any other direction, the outlined MEMS is a mono-axial accelerometer, having working range of $-50 /+50 \mathrm{~g}$. Anyway, during functioning other translations and rotations are not a priori obstructed by any structural constraints. As a consequence, during motion the main source of damping for such a system is essentially produced by two phenomena: the squeezed-film damping when the motion is predominant along the longitudinal direction normal to the electrodes, and the slide-film damping when the motion is predominant along a tangent direction.

\subsection{GOVERNING EQUATIONS AND SOLVING PROCEDURE}

The considered classical set of governing equations for elastodynamics study are reported below:

$$
M_{E E} \ddot{q}+C_{E E} \dot{q}+K_{E E} q=0
$$

where $\boldsymbol{M}_{\boldsymbol{E}}$ is the generalized mass matrix, $\boldsymbol{C}_{\boldsymbol{E}}$ is the damping matrix and $\boldsymbol{K}_{\boldsymbol{E}}$ is the stiffness matrix, as well as the 6-dimensional array $q$ of linear and rotational displacements of the reference point is introduced along with its first and second order time-derivatives. Let first introduce solving procedures adopted to compute mass and stiffness matrices. The generalized mass matrix was computed by two different approaches: the first one based on a lumped formulation in which all masses and inertials are considered to be located at in the center of mass of the proof mass (i.e. the central mass in our test case). The second one is otherwise based on a consistent distributed approach. We in fact recurred to a linearized formulation based on the Matrix Structural Analysis (MSA) for deriving the stiffness and inertia generalized matrices. In our assumptions the central body is considered as rigid while the blades are elastic elements modeled as flexible 3D-Euler beams. Small displacements are further taken into account. Flexible parts are split into beams and connected to the rigid central body by means of kinematic constraints. Boundary conditions are applied to the endbeams linked to the frame in order to consider the clamped connection. Details of the applied method can be found in [15].

Concerning the damping matrix determination, we used a modified Reynolds equation approach that allows to keep into account the damping effect due to squeezed-film as well as to the slide-film. It is to notice that application of that fluid-dynamical approach presumes accomplishment of the following assumptions: i) the fluid has a Newtonian, ideal and incompressible behavior; ii) the flow is laminar and isothermal; iii) inertia and body forces are negligible compared to the viscous and pressure forces iv) small variation of pressure are produced across the fluid film, so that they might be neglected; v) the fluid can be treated as a continuum and does not slip at the boundaries. The analytical expression of the modified Reynolds equation is reported below:

$$
\nabla_{t} \cdot\left(h^{3} Q_{c h} p \nabla_{t} p_{f}-6 \eta p h\left(\boldsymbol{u}_{t}+\boldsymbol{u}_{0, t}\right)\right)=12 \eta\left(\frac{d p f}{d t} h+p \frac{d h}{d t}\right)
$$

This equation allows to evaluate the film-pressure variation $p_{f}$, with respect to the ambient pressure $p$, in the gap between a mobile wall (electrodes' walls) and the channel base (mainframe ribs). The parameter $h$ is the height along a direction normal to the mobile wall, while $\boldsymbol{u}_{t}$ and $\boldsymbol{u}_{0, t}$ are the tangent velocity of the mobile structure and frame, respectively. The term $Q_{c h}$ is a function that takes into account gas rarefaction and relative flow-ratio. Damping 
matrix coefficients have been computed by running a coupled fluid-dynamical and structural numerical model in which the dumping effect is related to the squeezed-film pressure evaluated by numerically solving eq. (2), as better elucidated in the next section.

\subsection{NUMERICAL MODEL}

This section is devoted to present the numerical multi-physical FE model built up to solve the fluid-structure interaction, and to determinate by means of the procedure, discussed in the following, the damping matrix coefficients. We considered a coupled analysis in order to exploit the computed pressure fields by the fluid-dynamical analysis as input data for the structural simulation. On the other hand, structural properties and applied loads to the system determinate the fluid film pressure. Modelling and simulation has been carried-out by using the commercial FE-based software Comsol Multiphysics v3.5a [16]. The model was built-up considering a fixed- fixed constraints at the proof mass ends. From time to time, an impulsive force lying on the generic Cartesian axis, labeled as $x_{i}$, was applied at the center of mass of the proof mass. For each of these simulations, the inertial effect $-p d^{2} x_{i} / d t^{2}$ was considered along the same direction only. To all surfaces orthogonal to the chosen $x_{i}$ direction (represented in red in Fig. 2), we applied a resistant load whose value corresponds to the pressure force produced by the squeezed-film. Otherwise, all surfaces collinear to the $x_{i}$ direction were considered as free boundaries (green surfaces in Fig. 2) and a slide-film condition is applied for pressure field resolution. Once simulation is carried-out the damping coefficient for the generic $x_{i}$ translation is evaluated by applying the logarithmic decrement method to the displacement time history. The recursive application of this procedure for each translational direction allowed us to estimate the damping matrix coefficients, except for rotational terms that have been neglected in this study.

From a computational point of view, continuous equations were discretized on nostructured and no-uniform mesh made of tetrahedral Lagrange elements of order 2. Influence of spatial discretization has been preliminary checked, in order to assure mesh-independent results. Finally, a computational grid made giving up to 300,00 degrees of freedom for the system has been retained for computations. Time-marching was performed by adopting an Implicit Differential- Algebraic (IDA) solver [17], based on a variable-order and variablestep-size Backward Differentiation Formulas (BDF). Because the time-marching scheme is implicit, a nonlinear system of equations was solved each time step by applying a modified

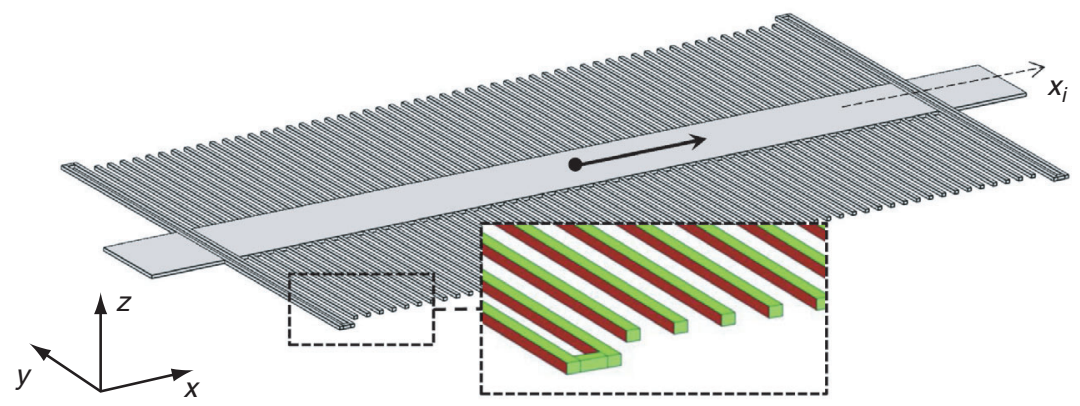

Figure 2 Graphical representation of applied impulsive force along the generic $x_{i}$ direction (black arrow), point of force application (black filled circle), distributed force/squeezed-film boundaries (red surfaces) and free/slide-film boundaries (green surfaces). 
Newton algorithm based on a discretized linearization by a first-order Taylor expansion. Algebraic systems of equations coming from differential operators discretization have been solved by a PARDISO package, a direct solver particularly efficient to solve unsymmetrical sparse matrixes by a LU decomposition technique.

\section{RESULTS}

In this section we present results related to the elastodynamic analysis, then we discuss the effect of the microchannels pressure on damping for the dynamic system. A comparison between mass matrix determination by using the lumped and distributed approach was firstly performed. The target has been to establish if the lumped approximation could be considered reliable in this kind of application. As introduced, the lumped model considers all the moving mass of the system located on the center of mass of the anchor body. Recurring to the parameters of Tab. I and denoting with $V_{1}$ and $V_{2}$ the volumes of the central body and one of the blade group, respectively, we obtain:

$$
m=\rho\left(V_{1}+2 V_{2}\right)=2.61 E-10[\mathrm{~kg}]
$$

The moments of inertia are calculated by the following expressions:

$$
\begin{aligned}
& l_{x}=\frac{1}{12} m\left(w^{2}+t^{2}\right) \\
& l_{y}=\frac{1}{12} m\left(L^{2}+t^{2}\right) \\
& l_{z}=\frac{1}{12} m\left(L^{2}+w^{2}\right)
\end{aligned}
$$

thereby, the generalized mass matrix $M_{\mathrm{EE}}$, as referred to the reference point, is defined as:

$$
\boldsymbol{M}_{E E}=\left[\begin{array}{cccccc}
2,6170 e-10 & 0 & 0 & 0 & 0 & 0 \\
0 & 2,6170 e-10 & 0 & 0 & 0 & 0 \\
0 & 0 & 2,6170 e-10 & 0 & 0 & 0 \\
0 & 0 & 0 & 5,4607 e-20 & 0 & 0 \\
0 & 0 & 0 & 0 & 5,0247 e-18 & 0 \\
0 & 0 & 0 & 0 & 0 & 5,0791 e-18
\end{array}\right]
$$

For the distributed approach, recurring to the expression of the mass matrix of a generic Euler beam and by means of FE method, the $3 \times 3$ matrix $\boldsymbol{M}_{E E^{*}}$ is finally derived, i.e.:

$$
\boldsymbol{M}_{E E^{*}}=\left[\begin{array}{ccc}
2,4930 e-10 & 0 & 0 \\
0 & 2,4927 e-10 & 0 \\
0 & 0 & 2,4930 e-10
\end{array}\right]
$$


In both cases, the computed off-diagonal entries of the mass matrix are null. Moreover, matrices cross-comparison shows the relative difference of diagonal terms is about $5 \%$, thus revealing the lumped approach is a good approximation for computing the mass matrix.

The computed stiffness matrix for the system is also reported below:

$$
\boldsymbol{K}_{E E}=\left[\begin{array}{cccccc}
6,4642 & 0 & 0 & 0 & 0 & -1,7764 e-15 \\
0 & 9,6368 & 0 & 0 & 0 & 4,6998 e-4 \\
0 & 0 & 2,4477 & 0 & -1,1937 e-4 & 0 \\
0 & 0 & 0 & 1,3497 e-8 & 0 & 0 \\
0 & 0 & -1,1937 e-4 & 0 & 1,5240 e-7 & 0 \\
-1,7764 e-15 & 4,6998 e-4 & 0 & 0 & 0 & 6,1462 e-7
\end{array}\right]
$$

Fig. 3-5 report Bode's diagrams evaluated in terms of magnitude and phase as a function of frequency. From figures it appears that the system is under-damped along the $\mathrm{x}$ - and $y$-directions, as revealed observing the peaks of the magnitude Bode's diagram, while it behaves as an over-dumped oscillator along the z-direction. The latter condition is worthwhile to prevent oscillations outside the plane of the accelerometer. Besides, reported diagrams agree with results presented in [14], where dynamics of a similar device has been investigated.

Influence of micro-channel pressure on damping has been also investigated. Following the procedure discussed in the previous section we applied impulsive force in FE model along each translational direction, run the simulation, recorded time-history of displacement along the same direction and then used the logarithmic decrement method to assess the corresponding term in the damping matrix. Fig. 6a-c graphically illustrates the applied procedure and results obtained for translation along $\mathrm{x}$ when pressure in the micro-channels is chosen to be $30 \mathrm{~Pa}$.

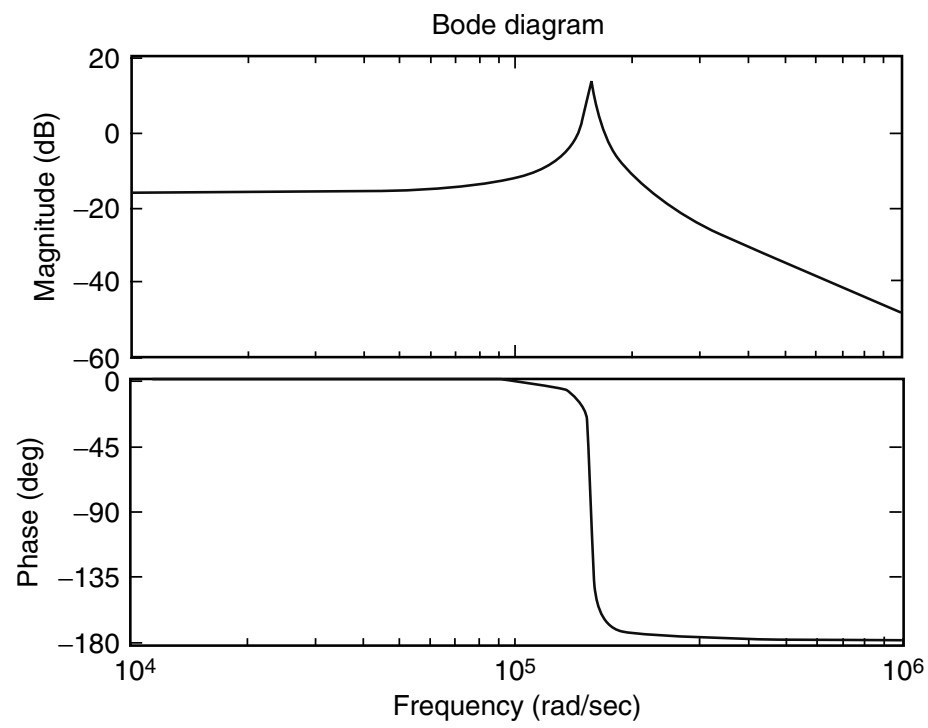

Figure 3 Bode diagram for the system along the $x$-direction. 


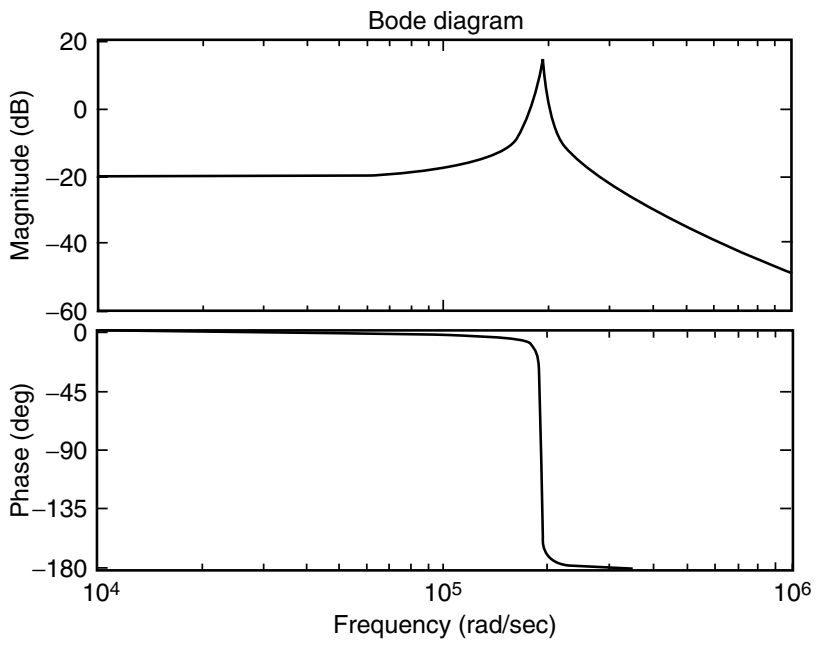

Figure 4 Bode diagram for the system along the $y$-direction.

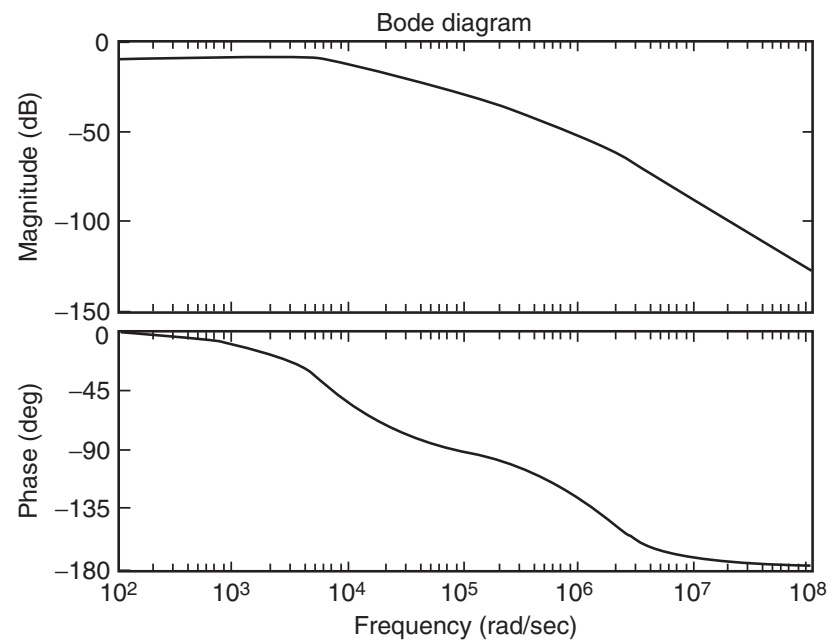

Figure 5 Bode diagram for the system along the $z$-direction.

The damping matrix for the different pressure values of air filling the microchannels are finally presented below:

$$
\boldsymbol{C}_{\mathbf{3 0}}=\left[\begin{array}{cccccc}
3,33 \cdot 10^{-5} & 0 & 0 & 0 & 0 & 0 \\
0 & 23,4 \cdot 10^{-5} & 0 & 0 & 0 & 0 \\
0 & 0 & 2,04 \cdot 10^{-6} & 0 & 0 & 0 \\
0 & 0 & 0 & 0 & 0 & 0 \\
0 & 0 & 0 & 0 & 0 & 0 \\
0 & 0 & 0 & 0 & 0 & 0
\end{array}\right]
$$




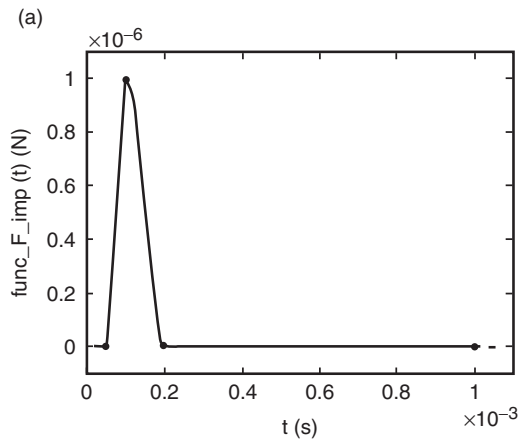

(b)

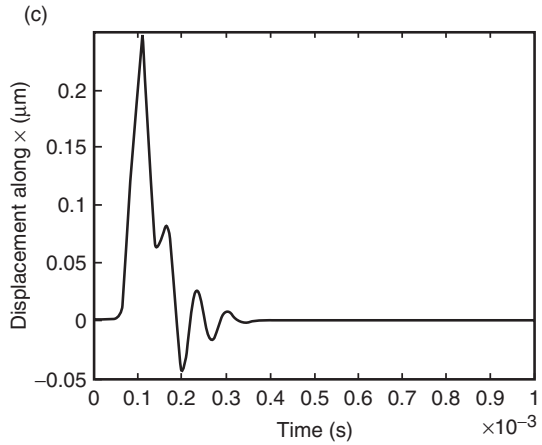

Figure 6 Impulsive force applied to the system, deformed system at a chosen time instant, time-history of displacement computed along the $x$-direction for $30 \mathrm{~Pa}$ of pressure in micro-channels.

$$
\begin{aligned}
\boldsymbol{C}_{\mathbf{3 0 0}} & =\left[\begin{array}{cccccc}
3,89 \cdot 10^{-5} & 0 & 0 & 0 & 0 & 0 \\
0 & 23,4 \cdot 10^{-5} & 0 & 0 & 0 & 0 \\
0 & 0 & 19,8 \cdot 10^{-6} & 0 & 0 & 0 \\
0 & 0 & 0 & 0 & 0 & 0 \\
0 & 0 & 0 & 0 & 0 & 0 \\
0 & 0 & 0 & 0 & 0 & 0
\end{array}\right] \\
\boldsymbol{C}_{\mathbf{3 0 0 0}} & =\left[\begin{array}{cccccc}
3,98 \cdot 10^{-5} & 0 & 0 & 0 & 0 & 0 \\
0 & 24,2 \cdot 10^{-5} & 0 & 0 & 0 & 0 \\
0 & 0 & 3,26 \cdot 10^{-4} & 0 & 0 & 0 \\
0 & 0 & 0 & 0 & 0 & 0 \\
0 & 0 & 0 & 0 & 0 & 0 \\
0 & 0 & 0 & 0 & 0 & 0
\end{array}\right]
\end{aligned}
$$

Where symbols $\boldsymbol{C}_{\mathbf{3 0}}, \boldsymbol{C}_{\mathbf{3 0 0}}$ and $\boldsymbol{C}_{\mathbf{3 0 0 0}}$ are used to indicate results computed for 30, 300 and $3000 \mathrm{~Pa}$, respectively. 


\section{CONCLUSIONS}

A numerical multi-physical investigation aimed to analyze system dynamics of an accelerometer has been carried-out. A coupled fluid-dynamical and structural FE model has been built up in order to compute the damping matrix for the system. Results presented in the form of Bode diagrams are in good agreement with reference data previously published concerning a similar device analysis. The proposed approach allowed to describe the accelerometer dynamics in the space, permitting to assess translational motion errors along directions apart the working one (x- direction). The proposed formulation can be used to analyze the dynamic behavior MEMs mechanisms based on compliant architectures so as to test different designs or new operative conditions without recurring to the real model. The simplicity of the method allows for the application of constrained optimization techniques computationally demanding for a commercial software.

\section{REFERENCES}

[1] Lobontiu, N., Compliant Mechanisms: Design of Flexure Hinges, CRC Press, 2003.

[2] Smith, S.T., Flexures: Elements of Elastic Mechanisms, Gordon and Breach Science Publishers, 2000.

[3] Simo, J.C. and Vu-Quoc, L., On the dynamics of flexible beams under large overall motions - The plane case: Part I, ASME Journal of Applied Mechanics, 1986, 53, 849-854.

[4] Simo, J.C. and Vu-Quoc, L., On the dynamics of flexible beams under large overall motions - The plane case: Part II, ASME Journal of Applied Mechanics, 1986, 53, 855-863.

[5] Shabana, A.A., Dynamics of multibody systems, Third Edition, Cambridge University Press, 2005.

[6] Lyon, S.M., Evans, M.S., Erickson, P.A. and Howell, L.L., 1997, Dynamic response of compliant mechanisms using the pseudo-rigid-body model: Proceedings of 1997 ASME Design Engineering Technical Conferences, DETC97/VIB-4177.

[7] Lyon, S.M., Evans, M.S., Erickson, P.A. and Howell, L.L., Prediction of the first modal frequency of compliant mechanism using the pseudorigid-body model, ASME J. Mech. Des., 1999, 121, 309-313.

[8] Yu, Y.Q., Howell, L.L., Lusk, C., Yue. Y. and He, M.G., Dynamic Modeling of Compliant Mechanisms Based on the Pseudo-Rigid-Body Model, Journal of Mechanical Design, 2005, 127, 760-765.

[9] Li, Z. and Kota, S., Dynamic Analysis of Compliant Mechanisms: Proceedings of the DETC'02 ASME 2002 Design Engineering Technical Conferences and Computer and Information in Engineering Conference, 2002, 1-10.

[10] Wang, W. and Yu, Y., New Approach to the Dynamic Modeling of Compliant Mechanisms, Journal of Mechanisms and Robotics, 2010, 2, 1-8.

[11] Lan, C.C., Lee, K.M. and Liou, J.H., Dynamics of Highly Elastic Mechanisms Using the Generalized Multiple Shooting Method: Simulations and Experiments, Mech. Mach. Theory, 2009, 44, 2164-2178.

[12] Lan, C.C. and Lee, K.M, Dynamic Model of a Compliant Link with Large Deflection and Shear Deformation: Proceedings of the 2005 IEEE/ASME International Conference on Advanced Intelligent Mechatronics, Monterey, California, USA, 2005.

[13] Przemieniecki, J.S., Theory of Matrix Structural Analysis, Dover Publications, Inc., New York, 1985.

[14] Cardou, P., Pasini, D. and Angeles, J., Lumped Elastodynamic Model for MEMS: Formulation and Validation, Journal of Microelectromechanical Systems, 2009, 17(4), 948-961.

[15] Cammarata, A., On the Stiffness Analysis and Elastodynamics of Parallel Kinematic Machines. In: Serial and Parallel Robot Manipulators: Kinematic Dynamics and Control, Kocaeli: Serdar Kucuk, 2012.

[16] Comsol Multiphysics v3.5a User's Guide, Comsol AB, 2008.

[17] Hindmarsh, A.C., Brown, P.N., Grant, K.E., Lee, S.L., Serban ,R., Shumaker, D.E. and Woodward, C.S., SUNDIALS: Suite of Nonlinear and Differential/Algebraic Equation Solvers. ACM Trans. Math. Software, 2005, 31, 363-396. 